\title{
Anillos vasculares: obstrucción de vía aérea en niños. Serie de casos
}

Vascular rings: airway obstruction in children. Case series

\author{
Dr. Adrián Zanetta ${ }^{a}$, Dra. Giselle Cuestas ${ }^{a}$, Dr. Hugo Rodríguez y y Dr. Carlos Tiscornia ${ }^{a}$
}

\section{RESUMEN}

En el niño, la compresión extrínseca de la tráquea es habitualmente de origen vascular, siendo menos frecuente la ocasionada por tumores, cardiopatías, quistes y abscesos.

Los anillos vasculares son anomalías congénitas del arco aórtico y sus ramas que comprimen la tráquea o el esófago en grado variable. Son poco frecuentes, pero constituyen una causa importante de dificultad respiratoria en el niño, por lo que se deben incluir en el diagnóstico diferencial de la obstrucción de la vía aérea superior.

Los síntomas de presentación son el estridor, la dificultad respiratoria y la disfagia de intensidad variable. El alto grado de sospecha clínica es el factor más importante para su diagnóstico, lo contrario, puede ocasionar un significativo retraso entre el inicio de los síntomas y el diagnóstico correcto.

Presentamos cuatro pacientes con diferentes tipos de anillos vasculares con el objetivo de describir manifestaciones clínicas, métodos diagnósticos y tratamiento.

Palabras clave: anillos vasculares, compresión traqueal.

\section{SUMMARY}

In children, extrinsic compression of the trachea is usually due to vascular origin, and less frequently caused by tumors, heart diseases, cysts and abscesses.

Vascular rings are congenital anomalies of the aortic arch and its branches that compress the trachea and/or esophagus to varying degrees. Although these congenital anomalies are not frequent, they constitute a major cause of respiratory distress in children. Thus, these anomalies should be included in the differential diagnosis of obstruction of the upper airway. Symptoms include stridor, respiratory distress and dysphagia of different intensity. The high degree of clinical suspicion is the most important factor for diagnosis, fail to do so can cause a significant delay between symptom onset and correct diagnosis. We present four patients with different types of vascular rings in order to describe clinical manifestations, diagnosis and treatment.

Key words: vascular rings, tracheal compression.

http:/ /dx.doi.org/10.5546/aap.2012.e110

a. Servicio de Endoscopia Respiratoria,

Hospital de Pediatría "Prof. Dr. Juan P. Garrahan".

Correspondencia:

Dr. Adrián Zanetta: adrianzanetta67@hotmail.com

Conflicto de intereses: Ninguno que declarar.

Recibido: 7-5-2012

Aceptado: 14-5-2012

\section{INTRODUCCIÓN}

Los anillos vasculares son anomalías o variantes anatómicas del arco aórtico que comprimen la tráquea o el esófago o ambos, y causa dificultades en la respiración o en la deglución. ${ }^{1}$ Su incidencia es baja; representa menos del 1\% de las anomalías vasculares congénitas. ${ }^{2,3}$ Son más frecuentes en varones $y$, en general, son anomalías aisladas. ${ }^{3}$ Más del 95\% de los anillos vasculares corresponden a uno de los siguientes: doble arco aórtico (DAA), arco derecho-ligamento izquierdo, compresión por arteria innominada y sling de la arteria pulmonar. ${ }^{1}$ Los dos primeros forman anillos completos, mientras que los restantes, llamados en la jerga "slings", no llegan a cerrar completamente un "anillo" vascular.

Los signos y síntomas se inician en los primeros meses de vida; son característicos: estridor bifásico o espiratorio que aumenta con el llanto y la alimentación, tos perruna, infecciones respiratorias recurrentes, episodios de apnea refleja y cianosis, sibilancias, hiperextensión cervical, retracción esternal e intercostal y dificultad en la alimentación, principalmente con la ingesta de sólidos. ${ }^{4-7}$

En la evaluación inicial de los niños con sospecha de anillos vasculares, el esofagograma y la endoscopia son útiles y las pruebas complementarias que confirman el diagnóstico son la angiotomografía (angioTC) o la angiorresonancia (angioRM) que determinan la causa de la compresión y proveen al cirujano cardiovascular la información para proceder a la corrección quirúrgica, de ser ésta necesaria.

\section{CASOS CLÍNICOS}

Presentamos cuatro pacientes con distintos tipos de anillos vasculares atendidos en el Servicio de Endoscopia Respiratoria del Hospital Garrahan entre diciembre de 2009 y diciembre de 2010.

En todos ellos se realizó radiografía cervical y de tórax, esofagograma con bario, traqueobroncoscopia bajo anestesia general y en ventilación espontánea, y angioTC. En tres pacientes se realizó angioTC con reconstrucción tridimensional (3D), y en uno, traqueobroncografía. El intervalo de edad 
al momento del diagnóstico fue de 2 a 18 meses.

Las características de los cuatro pacientes, incluidos síntomas, hallazgos endoscópicos y de los estudios por imágenes, y tratamiento, se resumen en la Tabla 1.

Tres pacientes recibieron tratamiento quirúrgico y la observación quirúrgica confirma los hallazgos tomográficos. La evolución posoperatoria ha sido satisfactoria.

\section{DISCUSIÓN}

Los anillos vasculares son el resultado del desarrollo anormal de los arcos aórticos fetales..$^{8-10}$ Rodean la tráquea y el esófago y frecuentemente los comprimen, por lo que causan dificultades en la respiración o la deglución. ${ }^{3,10}$

El anillo vascular completo más común es el DAA (casi el 70\%) y es el que provoca las compresiones traqueales más graves en el recién nacido.,4 Se forma a partir de la bifurcación de la aorta ascendente en dos arcos que rodean la tráquea y el esófago, y confluyen en la aorta descendente ( $F i$ gura 1). El sling que más frecuentemente produce compresión de la vía aérea es el del tronco braquiocefálico (Figura 2).

Si bien la incidencia de esta patología es baja, constituye una causa importante de obstrucción congénita de la vía aérea superior en niños, por lo que debe pensarse en ella, principalmente frente a tres situaciones: tos traqueal crónica o estridor bi-

TABLA 1. Pacientes con anillo vascular.

\begin{tabular}{|c|c|c|c|c|c|c|c|c|}
\hline$\underline{\mathbf{P}}$ & Sexo & Edad al diagnóstico & Síntomas & Esofagograma & Endoscopia & AngioTC & Malf asociadas & $s$ Tratamiento \\
\hline 1 & M & 7 meses & $\begin{array}{l}\text { Estridor bifásico, } \\
\text { dificultad respiratoria y } \\
\text { en deglución de sólidos, } \\
\text { crisis de cianosis, } \\
\text { infecciones respiratorias } \\
\text { recurrentes } \\
\end{array}$ & $\begin{array}{l}\text { Compresión } \\
\text { posterior y } \\
\text { lateral }\end{array}$ & $\begin{array}{l}\text { Compresión } \\
\text { traqueal } \\
\text { AP del } 80 \%\end{array}$ & $\begin{array}{l}\text { Doble } \\
\text { arco } \\
\text { aórtico } \\
\text { - }\end{array}$ & 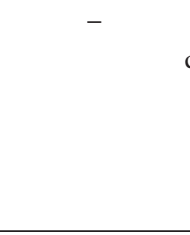 & $\begin{array}{c}\text { Sección } \\
\text { de arco aórtico } \\
\text { menor } \\
\text { y del } \\
\text { ligamento } \\
\text { arterioso } \\
\end{array}$ \\
\hline 2 & M & 2 meses & $\begin{array}{l}\text { Estridor bifásico, } \\
\text { tos crónica, } \\
\text { crisis de cianosis }\end{array}$ & Normal & $\begin{array}{l}\text { Compresión } \\
\text { traqueal } \\
\text { ALD del } 70 \%\end{array}$ & $\begin{array}{c}\text { Arteria } \\
\text { innominada } \\
\text { aberrante }\end{array}$ & a & $\begin{array}{l}\text { Arteriopexia } \\
\text { a pared posterior } \\
\text { del esternón } \\
\end{array}$ \\
\hline 3 & M & 12 meses & $\begin{array}{c}\text { Estridor bifásico, } \\
\text { dificultad respiratoria, } \\
\text { crisis de cianosis, } \\
\text { tiraje }\end{array}$ & $\begin{array}{l}\text { Muesca } \\
\text { anterior }\end{array}$ & Microtráquea & $\begin{array}{c}\text { Sling } \\
\text { de la arteria } \\
\text { pulmonar }\end{array}$ & Microtráquea & $\begin{array}{l}\text { Deslizamiento } \\
\text { traqueal } \\
\text { por detrás } \\
\text { de arteria } \\
\text { pulmonar } \\
\end{array}$ \\
\hline \pm & M & 18 meses & $\begin{array}{l}\text { Disfagia } \\
\text { para sólidos, } \\
\text { tos crónica }\end{array}$ & $\begin{array}{c}\text { Compresión } \\
\text { posterior }\end{array}$ & $\begin{array}{l}\text { Compresión } \\
\text { traqueal AP } \\
\text { del } 60 \%\end{array}$ & $\begin{array}{l}\text { Arco aórtico } \\
\text { derecho } \\
\text { arteria subclavi } \\
\text { quierda aberrar }\end{array}$ & $\begin{array}{l}\text { VACTERL } \\
\text { ia } \\
\text { inte }\end{array}$ & $\begin{array}{l}\text { Conservador } \\
\text { (alto riesgo } \\
\text { quirúrgico) }\end{array}$ \\
\hline
\end{tabular}

P: pacientes; AngioTC: angiotomografía; Malf: malformaciones; M: masculino; AP: anteroposterior; ALD: anterolateral derecha; art: arteria; izq: izquierda; VACTERL: defectos vertebrales, ano imperforado, malformaciones cardiovasculares, fístula tráqueoesofágica con atresia de esófago o sin ella, displasia renal, polidactilia y deformidades de los miembros.

Figura 1. Doble arco aórtico. A. Esofagograma. B. Endoscopia. C. AngioTC 3D.

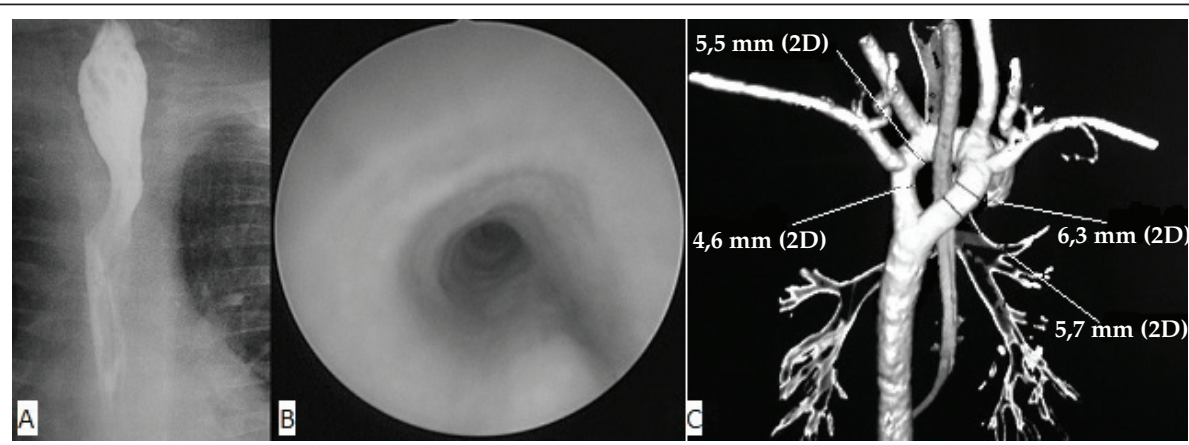


fásico persistente, síndrome bronquiolítico que no responde al tratamiento broncodilatador, y frente a fallas en la extubación.

La clínica, similar en los distintos tipos de anillos vasculares, se inicia durante las primeras semanas de vida. ${ }^{3}$ Los síntomas respiratorios, sobre todo el estridor, son los más frecuentes al momento del diagnóstico. ${ }^{6}$ Sin embargo, los anillos vasculares son responsables de una pequeña proporción de los lactantes con respiración ruidosa, la mayoría de los cuales tiene laringomalacia. ${ }^{2}$ Una clave clínica en la diferenciación es que el estridor en los anillos vasculares es tanto espiratorio cuanto inspiratorio.

La investigación inicial en niños con sospecha de anillo vascular debe incluir un esofagograma con bario que muestra las indentaciones esofágicas. Presenta un rendimiento elevado ( $>95 \%)$, pero en la compresión por la arteria innominada es normal. ${ }^{3}$

En la radiografía cervical se puede observar la disminución del calibre traqueal. La radiografía de tórax simple posee un valor relativo. ${ }^{3}$
La laringotraqueobroncoscopia permite documentar la compresión pulsátil de la vía aérea y descartar otras causas obstructivas con estridor (laringomalacia, parálisis cordal, angioma subglótico, traqueomalacia). ${ }^{5,11}$ La endoscopia virtual se presenta como una alternativa, pero es una exploración estática que tendría limitaciones en la evaluación de la obstrucción dinámica. Puede dar lugar a artificios debido a la retención de moco distal a la obstrucción y no provee información sobre la calidad de la mucosa traqueal. ${ }^{12}$

Los exámenes complementarios que confirman el diagnóstico son la angioTC o la angioRM. A diferencia de la angiografía, no son invasivas. ${ }^{1,3,6}$ Permiten el diagnóstico diferencial con otras causas de compresión traqueal extrínseca: tumores del mediastino, quistes broncogénicos, hemangiomas, entre otros. ${ }^{6}$

La combinación de evaluación endoscópica y angioTC resultó ser una estrategia diagnóstica eficaz en nuestros pacientes.

Figura 2. Compresión traqueal por arteria innominada. A. Endoscopia. B. AngioTC 3D.

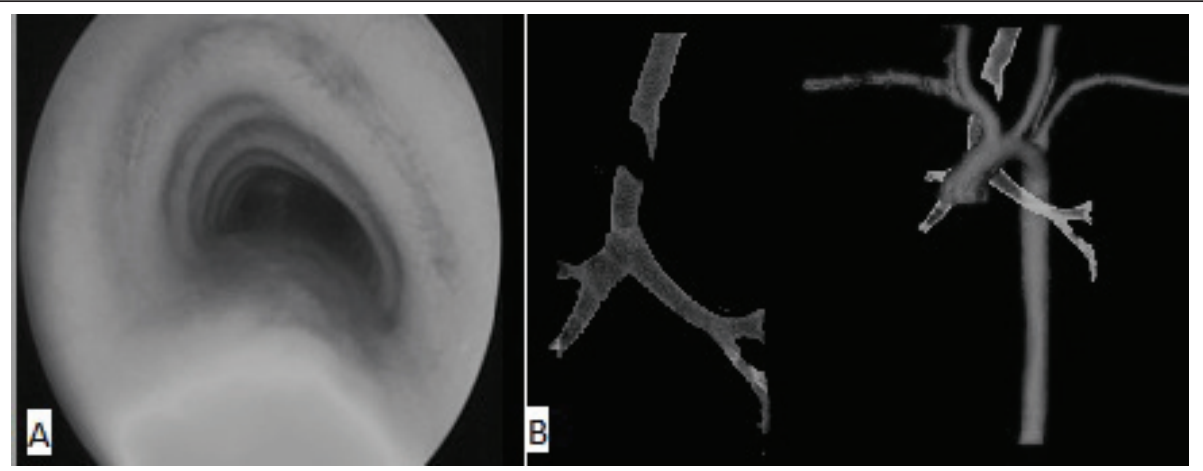

FIGURA 3. Sling de la pulmonar. A. Endoscopia: anillos traqueales completos. B. TC: la rama izquierda de la arteria pulmonar se origina de la derecha, y cruza por detrás a la tráquea (y por delante del esofágo) hacia el pulmón izquierdo.
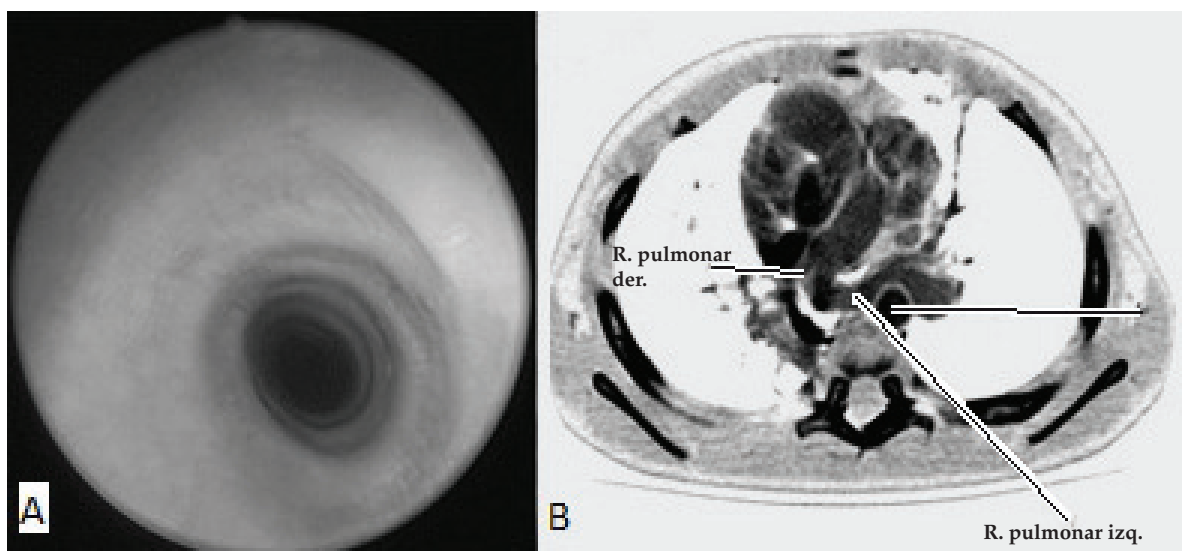
El tratamiento dependerá de la gravedad de los síntomas. El manejo inicial se basa en una adecuada fisioterapia respiratoria, antibioticoterapia precoz de las infecciones respiratorias, aerosoles humidificadores, incluso oxigenoterapia y curas cortas de corticosteroides. ${ }^{4,7}$

El tratamiento quirúrgico está indicado en quienes presentan sintomatología evidente (episodios de apneas y cianosis, neumonías a repetición), en los rebeldes al tratamiento conservador con compresión de vía aérea mayor del $50 \%$, y ante la imposibilidad de extubación. ${ }^{5,7}$ La corrección quirúrgica es eficaz y el riesgo de la intervención es mínimo. ${ }^{13}$

La mayoría de los pacientes con compresión por arteria innominada mejora de manera espontánea entre los 12 y 24 meses de edad. Si es necesario tratamiento quirúrgico, la técnica de elección es la aortopexia (o arteriopexia innominada), que consiste en la fijación de la cara anterior del cayado aórtico (o arteria innominada) a la pared posterior del esternón. ${ }^{4,14}$

En el caso de doble arco aórtico la cirugía consiste en la sección del arco aórtico menor y en el sling de la arteria pulmonar corresponde situar la arteria pulmonar izquierda por delante de la tráquea y resolver la estenosis traqueal, si existe. ${ }^{14}$ Un tercio de los niños con sling de la pulmonar presentan asociado un segmento de anillos traqueales completos, como nuestro paciente, siendo la lesión más importante, a menudo, la obstrucción intrínseca. ${ }^{14}$ (Figura 3).

En los niños con anillos vasculares, el compromiso de la vía aérea es por compresión directa externa y por el desarrollo subsecuente de malacia. ${ }^{6}$ En todos los casos, cuando la compresión puede ser eliminada, es frecuente que la sintomatología de traqueomalacia, generalmente mínima, persista una vez retirado el obstáculo y se atenúe progresivamente. Una sintomatología postoperatoria de traqueomalacia grave debe llevar a realizar una reevaluación endoscópica de la vía aérea. ${ }^{4,15}$

En conclusión, los anillos vasculares son anomalías vasculares infrecuentes. Para su diagnóstico precoz es necesaria una alta sospecha clínica. Los pacientes con síntomas respiratorios recurrentes (como tos crónica, estridor y sibilancias) deben ser examinados por la posible presencia de anillos vasculares. La endoscopia es un arma diagnósti- ca importante para evaluar niños con estridor. El esofagograma presenta una alta sensibilidad, requiriéndose una angioTC o angioRM para precisar la anatomía del anillo vascular.

\section{BIBLIOGRAFÍA}

1. Ma GQ, Li ZZ, Li XF, Peng Y, et al. Congenital vascular rings: a rare cause of respiratory distress in infants and children. Chin Med J 2007;120:1408-12.

2. Thomson AH, Beardsmore CS, Firmin R, Leanage R, et al. Airway function in infants with vascular rings: preoperative and postoperative assessment. Arch Dis Child 1990;65:171-4.

3. Vera de Pedro E, Martínez Ayúcar M, Marín Gonzalo A, Galdeano Miranda JM, et al. Anillos vasculares completos. An Pediatr 2008;69:52-5.

4. Froehlich P, Furminieux V, Fontaine P, Morgon A. Anomalías congénitas de la tráquea y de los grandes bronquios. Encycl Med Chir. Otorhinolaryngologie 20-770-A-10, 2000: 1-9.

5. Holinger LD, Green CG, Benjamin B, Sharp JK. Tracheobronchial Tree. En: Holinger LD, Lusk RD, Green CG, eds. Pediatric Laryngology and Bronchoesophagology. Filadelfia: Lippincott-Raven; 1997.Págs.187-213.

6. Malik TH, Bruce IA, Kaushik V, Willatt DJ, et al. The role of magnetic resonance imaging in the assessment of suspected extrinsic tracheobronchial compression due to vascular anomalies. Arch Dis Child 2006;91:52-5.

7. Sommer D, Forte V. Avances en el tratamiento de colapso de vías respiratorias mayores. Férulas en vías respiratorias. En: Jong AL, Kuppersmith RB, eds. Clínicas otorrinolaringológicas de Norteamérica. Actualización en vías respiratorias de niños. México; 2000.Págs.155-69.

8. Becit N, Erkut B, Karaca Y. Vascular ring. Tracheoesophageal compression associated with symmetrical double aortic arch. Tex Heart Inst J 2008;35:208-10.

9. Mainwaring RD, Jacobson JL, Reinhartz O, Gates RL. Unusual manifestations of vascular rings. J Card Surg 2010;25:79-83.

10. Subramanyan R, Venugopalan P,Narayan R.Vascular Rings: An important Cause of Persistent Respiratory Symptoms in Infants and Children. Indian Pediatr 2003;40:951-7.

11. Gaafar AH, El-Noueam KI. Bronchoscopy versus multidetector computed tomography in the diagnosis of congenital vascular ring. J Laryngol Otol 2011;125:301-8.

12. Monnier P. Clinical evaluation of airway obstruction. En: Pediatric Airway Surgery. Lausana: Springer; 2011. Págs.31-44.

13. Bové T, Demanet H, Casimir G, Viart P, et al. Tracheobronchial compression of vascular origin. Review of experience in infants and children. J Cardiovasc Surg 2001;42:663-6.

14. Andrews TM, Myer CM, Bailey WW, Vester SR. Intrathoracic Lesions Involving the Tracheobronchial Tree. En:Myer CM, Cotton RT, Shott SR, eds. The Pediatric Airway. An Interdisciplinary Approach. Filadelfia: Lippincott; 1995. Págs.223-45.

15. Fleck RJ, Pacharn P, Fricke BL, Ziegler MA, et al. Imaging findings in pediatric patients with persistent airway symptoms after surgery for double aortic arch. AJR 2002;178:1275-9. 